\title{
Comparative Thermodynamic Properties of the Restricted Rotator and the Harmonic Oscillator
}

\author{
J. O. HALFORD \\ Department of Chemistry, University of Michigan, Ann Arbor, Michigan
}

(Received March 10, 1947)

\begin{abstract}
The differences between the thermodynamic properties of the restricted internal rotator and a defined harmonic oscillator are expressed within narrow limits as a function of the single variable $V / R T$, where $V$ is the height of the restricting potential barrier. As a result, a single table with one column for each of the four thermodynamic functions can be used in conjunction with standard harmonic oscillator tables to give the restricted rotator properties. This approximation is accurate within $0.02 \mathrm{cal} / / \mathrm{mole} / \mathrm{deg}$. for nearly all cases to which it might be applied, and it is shown to be improbable that rotators will be found for which the error would rise appreciably above this limit. The harmonic oscillator chosen for comparison has a slightly lower frequency than the limiting one approached by the restricted rotator at very high values of $V / R T$.
\end{abstract}

$I^{\mathrm{N}}$ $\mathrm{N}$ connection with another problem the writer had occasion to make use of the tables of thermodynamic properties compiled by Pitzer ${ }^{1}$ and by Pitzer and Gwinn ${ }^{2}$ for the restricted internal rotator, and to look up a number of cases in which the restricting potential barrier has been evaluated from thermodynamic data with the aid of the entropy tables. With very few exceptions the barriers are above $2500 \mathrm{cal} . / \mathrm{mole}$, so that at ordinary and lower temperatures few molecules can acquire enough energy to rotate. Consequently, these systems can be regarded as vibrators possessing rather large anharmonicities.

It therefore seemed desirable to look into the question of tabulating the thermodynamic properties of the restricted rotator in relation to the harmonic oscillator rather than the free rotator, thus emphasizing the essentially oscillatory character of the motion and providing an alternative method of calculation.

When the differences between the thermodynamic properties of the restricted rotator and the limiting harmonic oscillator for which

$$
h \nu / k T=\left(1 / Q_{f}\right)(\pi V / R T)^{\frac{1}{2}}
$$

are examined, they prove to be small except at very low $V / R T$. They are also almost independent of $Q_{f}$, so that within limits of the order of $0.05 \mathrm{cal} . / \mathrm{mole} / \mathrm{deg}$, these differences depend only upon $V / R T$. This is in accord with the

\footnotetext{
${ }^{1}$ K. S. Pitzer, J. Chem. Phys. 5, 469 (1937).

${ }^{2}$ K. S. Pitzer and W. D. Gwinn; J. Chem. Phys. 10, 428 (1942).
}

relation proposed by Pitzer and Gwinn under the heading "A Useful Approximation," for which they give the equation

$$
Q=Q_{\text {olass }}\left(Q_{\text {Ho, quant }} / Q_{\text {HO, class }}\right) \text {. }
$$

$Q$ is the partition function of the restricted rotator, $Q_{\text {Ho }}$ that of the harmonic oscillator, and the subscript quant or class refers to the quantum mechanical or classical variety of function. In Eq. (1), $Q_{f}$ is the partition function of the free rotator and $V$ is the height of the potential barrier or the maximum potential energy of the rotational degree of freedom.

Equation (2) implies that the ratio $Q / Q_{\text {Ho }}$ at a given value of $V / R T$ is independent of $Q_{f}$ and remains equal to its limiting or classical value. It follows that the differences between the thermodynamic functions should be similarly independent of $Q_{f}$. It is in this form that the approximation is accurate to about $0.05 \mathrm{cal} . / \mathrm{mole} / \mathrm{deg}$. in the useful range of the variables. The error is somewhat less, as originally shown by Pitzer and Gwinn, for values of $1 / Q$, below 0.4 , but it becomes larger when $1 / Q_{f}$ is high, even at the highest values of $V / R T$.

Two devices may be used to improve the accuracy of the approximation. The first is to change the frequency of the harmonic oscillator by substituting another constant for $\pi^{\frac{1}{3}}$ in Eq. (1). This change will affect the entropy and free energy more than the relatively insensitive heat content and heat capacity. The second is to tabulate at each value of $V / R T$ an average dif- 
ference rather than the limiting classical one, thereby cutting the maximum error approximately in half.

Exactly what harmonic oscillator is most suitable depends upon which of the thermodynamic functions is to be represented most accurately. Here the emphasis has been placed upon the entropy, for which 1.74 or 1.75 can be substituted for $\pi^{\frac{1}{3}}$ in Eq. (1) with about equally good results.

Table $I$ shows the averaged increments to be added to the thermodynamic properties of the harmonic vibrator

$$
h \nu / k T=\left(1.74 / Q_{f}\right)(V / R T)^{\frac{1}{3}}
$$

to give the corresponding properties of the restricted rotator. Each row shows, consecutively for a given $V / R T$, the entropy, free energy, heat content, and heat capacity increments.

\section{ACCURACY}

The differences shown in Table $I$ are in general consistent with the extended tables of Pitzer and Gwinn to within $0.01 \mathrm{cal} . / \mathrm{mole} / \mathrm{deg}$. Interpolation in the least favorable regions should not increase this uncertainty beyond 0.02 . For each value of $V / R T$, however, there is a limit to $1 / Q_{f}$ beyond which the approximation becomes less accurate. When $V / R T$ is high, the Pitzer and Gwinn tables are beginning to show similar uncertainties, but at low $V / R T$ the larger deviations may appear well within the region in which the properties are accurately known. No attempt will be made to describe how the accuracy falls off under these circumstances because it can be shown that the region of large errors probably lies beyond the limit of useful application of the table.

In the region in question the reduced moment of inertia is low, and $V / R T$ is relatively low. Since there are no molecules with symmetrical tops having reduced moments less than that of methanol, the methanol moment, a little above $1.0 \times 10^{-40}$ c.g.s. units, will set an upper limit to the error. From Pitzer and Gwinn,

$$
Q_{f}=(2.815 / n)\left(10^{38} I T\right)^{\frac{1}{2}} \text {. }
$$

For $10^{40} I=1.0$ and $n=3, Q_{f}=0.094 T^{t}$. A temperature is now chosen and for the resulting $Q_{f}$ the value of $V / R T$ is estimated below which the
TABLE I. Comparative thermodynamic properties of the restricted rotator and the harmonic oscillator of Eq. (3).

\begin{tabular}{rrrrr}
\hline$V / R T$ & $S-S_{H}$ & $-\left(F-F_{H}\right) / T$ & $\left(H-H_{H}\right) / T$ & \multicolumn{1}{c}{$C-C_{H}$} \\
\hline 0.6 & -0.45 & 0.03 & -0.48 & -0.90 \\
1.0 & -0.02 & .22 & -0.24 & -0.76 \\
2.0 & 0.37 & .27 & 0.10 & -0.29 \\
3.0 & .41 & .20 & .21 & 0.10 \\
4.0 & .35 & .14 & .21 & .30 \\
6.0 & .21 & .08 & .13 & .32 \\
9.0 & .11 & .03 & .08 & .19 \\
12.0 & .06 & .02 & .04 & .12 \\
16.0 & .04 & .01 & .03 & .07 \\
20.0 & .02 & .0 & .02 & .05 \\
\hline
\end{tabular}

error in the free energy exceeds 0.02 . This will give the minimum potential barrier which would permit calculation of the free energy within this limit. Table II shows the result of this estimate.

In round figures, it appears that a potential barrier above $2000 \mathrm{cal}$./mole will permit accurate calculation. The situation is about the same with the heat content and heat capacity and is considerably more favorable with the entropy. It is probable that the correct barrier for methanol is at least this high. For all other organic molecules the limiting barrier will be lower. The case of dimethylacetylene should perhaps be mentioned because the potential barrier is low. Here the limiting barrier for accurate calculation is about $1000 \mathrm{cal} . / \mathrm{mole}$, but the question of an approximate calculation is less important because the internal rotation in this case is almost free.

It is interesting that this consideration also shows that the region in which the Pitzer and Gwinn tables are beginning to show inaccuracies lies outside the probable limit of usefulness.

\section{USE OF TABLE I}

When the temperature and the potential barrier are known, a thermodynamic property can be quickly evaluated. The ratio $h \nu / k T$ is determined from Eq. (3), and the property is obtained for the harmonic oscillator from standard tables such as those in Mayer and

TABLE II. Minimum potential barrier for calculation of the free energy within 0.02 unit at $10^{40} I=1.0$.

\begin{tabular}{cccc}
\hline$T$ & $1 / Q_{f}$ & $V / R T$ (min.) & $V$ (min.) \\
\hline 900 & 0.354 & 0.7 & 1250 \\
625 & .427 & 1.5 & 1860 \\
400 & .532 & 2.0 & 1600 \\
225 & .708 & 3.5 & 1600 \\
\hline
\end{tabular}


TABle III. Potential barrier for ethane. $10^{40} I=2.7$ and $S=1.70$ at $T=298$.

\begin{tabular}{ccccc}
\hline Approx. & $\delta S$ & $h \nu / k T$ & $V / R T$ & $\delta S$ (new) \\
\hline 1 & 0.20 & 1.378 & 4.44 & 0.32 \\
2 & .26 & 1.428 & 4.77 & .30 \\
3 & .28 & 1.445 & 4.886 & .288 \\
4 & .284 & 1.448 & 4.906 & .286 \\
\hline
\end{tabular}

Mayer ${ }^{3}$ Addition of the increment from Table I for the correct level of $V / R T$ gives the approximate value of the property for the restricted rotator.

For example, take $V / R T=3.3$ and $1 / Q_{f}=0.38$. Then $h \nu / k T=1.201$ and $S_{H}=0.876 R=1.742$. Add 0.39 from Table I for a total entropy of 2.132. From Pitzer and Gwinn, Table III, a double linear in terpolation gives the value 2.138.

To determine the height of the potential barrier from the entropy requires a series of approximations, since both the barrier and the increment from Table I depend upon $V / R T$. For ethane, take $10^{40} I=2.7$ and $S=1.70$ at $T=298$. This gives $1 / Q_{f}=0.376$. First an average increment of 0.20 from the entropy column of Table I is subtracted, leaving 1.50 for the harmonic oscillator and giving $h \nu / k T=1.38$. Then $V / R T$ $=\left(Q_{f} / 1.74\right)^{2}(h \nu / k T)^{2}=2.340(h \nu / k T)^{2}=4.44$. This yields a new correction from Table $I$ and the calculation is repeated. Rapid convergence is obtained by taking for each new approximation the numerical mean of the newly indicated increment and the one just previously used. The approximations are summarized in Table III.

The result is $V / R T=4.92$ and $V=2913$ cal./mole. From Table III of Pitzer and Gwinn, again by means of a double linear interpolation, for $S=1.70$ and $1 / Q_{f}=0.376, V / R T=4.934$ and $V=2921$.

The approximation yields results almost as good as the extended tables of Pitzer and Gwinn and appears to be accurate enough for use with any data now available or in prospect. The calculation, however, is not always as convenient as could be desired, but in compensation has the advantage of emphasizing that the thermodynamic properties of the restricted rotator are related in a simple way to those of the harmonic oscillator.

${ }^{3}$ Mayer and Mayer, Statistical Mechanics (John Wiley and Sons, Inc., New York, 1946), p. 445.

\section{APPENDIX}

Setting up an approximate method of reproducing the values in the Pitzer and Gwinn tables implies dependence upon the potential energy function $V=V_{0}(1-\cos n \theta) / 2$. The potential barrier is now represented by $V_{0}$ and $n$ is the symmetry number of the number of potential minima in the cycle. $\theta$ is a combination coordinate in which a single rotator of moment of inertia $I$, equal to the reduced moment of the internal rotator, would have the same properties as the rotator under consideration.

Since $\cos n \theta=1-n^{2} \theta^{2} / 2+\cdots$, we may write for small values of $\theta, \quad V=V_{0} n^{2} \theta^{2} / 4$ for the limiting harmonic or parabolic potential energy at high levels of $V / R T$. The corresponding force constant is $V_{0} n^{2} / 2$ and, consequently, $h \nu / k T$ $=(n h / 2 \pi k T)\left(V_{0} / 2 I\right)^{\frac{1}{2}}$. When this expression is combined with $Q_{f}=\left(8 \pi^{3} I k T\right)^{\frac{1}{2}} / n h$, we obtain Eq. (1).

To calculate the increments of Table I, a set of frequencies according to Eq. (3) was first tabulated as a function of $V / R T$ and $1 / Q_{f}$. The four thermodynamic properties of the harmonic oscillator were then tabulated in the same framework. Next, those parts of the entropy and free energy tables which are given only as deficiencies below free rotation were converted, except at $1 / Q_{f}=0$, to read these properties directly. For this purpose, $F_{f}=R T \ln \left(1 / Q_{f}\right)$ and $S_{f}=R / 2-F_{f} / T$. The differences between harmonic oscillator and restricted rotator could then be tabulated and used to find the increments shown in Table I. The gas constant $R$ has been taken as 1.9869 .

At $1 / Q_{f}=0$, the limiting ratio

$$
Q_{f} / Q_{H}=1.74(V / R T)^{\frac{1}{2}}
$$

was used in conjunction with the tabulated differences $S_{f}-S$ and $\left(F-F_{f}\right) / T$ to find $S-S_{H}$ and $\left(F_{H}-F\right) / T$.

Finally, the effects produced by changing the constant $a$ in the equation

$$
h \nu / k T=\left(a / Q_{f}\right)(V / R T)^{\frac{1}{2}}
$$

will be considered. For the harmonic oscillator,

and

$$
F_{H} / T=R \ln \left(1-e^{-k \nu / k \cdot T}\right),
$$

$$
H_{H} / T=(R h \nu / k T) /\left(e^{h v / k T}-1\right) .
$$


A small change in $a$ at constant $Q_{f}$ and $V / R T$ affects these functions as follows:

and

$$
\delta\left(F_{H} / T\right) / \delta a=H_{H} / a T
$$

$$
\delta\left(H_{H} / T\right) / \delta a=H_{H} / a T-C_{H} / a .
$$

Since $S=(H-F) / T$, it follows that

$$
\delta S_{H} / \delta a=-C_{H} / a
$$

Thus the changes in the harmonic oscillator properties, and therefore also in the differences of Table 1 , are related in a simple way to the properties themselves. At $1 / Q_{f}=0$, a given small change in $a$ will produce the same effect upon the entropy and free energy at all values of $V / R T$, while at the same time the heat content will remain unchanged at the classical value. When $V / R T$ is low, the frequency remains low through the whole useful range of $1 / Q_{f}$, and the effect of changing $a$ will change only slightly as $1 / Q_{f}$ increases. If $V / R T$ is high, a change in $a$ will change the entropy and free energy by amounts varying from zero at high $1 / Q_{f}$ to $R$ times the percentage change in $a$ at low $1 / Q_{f}$.

This shows how the trend of 0.05 to 0.06 in the entropy and free energy differences found for the limiting harmonic oscillator at high $V / R T$ can be effectively leveled out by the small change in $a$ from $\pi^{\frac{1}{2}}$ to 1.74 . The effect on the heat content and heat capacity is zero at both ends of the $1 / Q_{f}$ scale and reaches a low maximum at some intermediate but fairly high $1 / Q_{f}$. This maximum is less than one-half the maximum effect on the entropy and free energy.

It is reasonable that the most suitable harmonic oscillator for the comparison should have frequencies below the limiting ones, because in the useful range of the variables $R T$ is always an appreciable fraction of $V$ and, as a consequence, the average spacing of the more important energy levels will be less than that of the limiting oscillator.

\title{
Intermolecular Forces and Energies of Vaporization of Liquids
}

\author{
SIDNEY W. BENSON \\ Chemical Laboratories, University of Southern California, Los Angeles, California
}

(Received April 4, 1947)

By using a "smoothed potential" model for the liquid state and assuming isotropic, homogeneous expansion with temperature which involves no change in coordination, it is shown that it is possible to reduce the expression for the configurational energy of the liquid to a function of a single parameter, the density. If the intermolecular forces can be put into a polynomial form then the expression for the configurational energy can be factored, resulting in an algebraic equation of the form:

$$
E_{c}=-B_{1} D^{2}-B_{2} D^{8 / 3}-B_{3} D^{-10 / 3}+B_{4} D^{4}
$$

\section{INTRODUCTION}

$I^{N}$ $\mathrm{N}$ recent years, the problem of expressing analytically the thermodynamic properties of

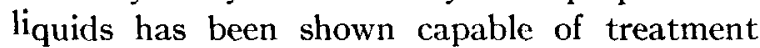
using the methods of statistical mechanics. The most general and rigorous treatment made by Mayer and other workers, ${ }^{1}$ has thrown consider-

${ }_{1}$ Mayer and Mayer, Statistical Mechanics, Chaps. 13, 14 (John Wiley, 1940) (summarizes most of the recent work); in which $D$ is the molar density and the $B$ 's are constants independent of temperature. For the distances occurring in liquids, the last three terms can be either dropped or combined with the first, leaving as an approximation: $E_{c}=A D^{x / 3}$. The energy of vaporization is given by: $E_{v}=A\left(D_{1}^{x / 3}-D_{q}^{x / 3}\right)$. Values calculated from this last equation show excellent agreement with experimental values for a large number of both normal and "abnormal" liquids. Values of $x=5$ or 6 both work, the latter being better up to the critical temperature.

able light on the phenomenon of condensation and raised fundamental questions concerning the region of the critical temperature. However, while fundamental in its approach, this method has not yielded functions susceptible to a simple, practical treatment.

Simpler models of the liquid state, based on see also: Band et al., J. Chem. Phys. 9, 123 (1941) and earlier papers. 